山्山FARAÇAISE

$\gg \mathrm{DE}$

핌AGOGIE

\section{Revue française de pédagogie}

Recherches en éducation

172 | juillet-septembre 2010

La pédagogie universitaire : un courant en plein développement

\title{
METTON-GAYON Céline. Les adolescents, leur téléphone et Internet. " Tu viens sur MSN? »
}

Paris : L'Harmattan, 2009, 202 p.

\section{Anne Barrère}

\section{(2) OpenEdition \\ Journals}

Édition électronique

URL : http://journals.openedition.org/rfp/2322

DOI : $10.4000 /$ rfp.2322

ISSN : 2105-2913

Éditeur

ENS Éditions

Édition imprimée

Date de publication : 15 juillet 2010

Pagination : 143-144

ISBN : 978-2-7342-1188-4

ISSN : 0556-7807

Référence électronique

Anne Barrère, «Metton-gayon Céline. Les adolescents, leur téléphone et Internet. « Tu viens sur MSN ? » », Revue française de pédagogie [En ligne], 172 I juillet-septembre 2010, mis en ligne le 24 janvier 2011, consulté le 22 septembre 2020. URL : http://journals.openedition.org/rfp/2322 ; DOI : https://doi.org/10.4000/rfp.2322

Ce document a été généré automatiquement le 22 septembre 2020.

(c) tous droits réservés 


\title{
METTON-GAyON Céline. Les adolescents, leur téléphone et Internet. « Tu viens sur MSN ?»
}

Paris : L'Harmattan, 2009, 202 p.

\author{
Anne Barrère
}

\section{RÉFÉRENCE}

METTON-GAYON Céline. Les adolescents, leur téléphone et Internet. « Tu viens sur MSN ? »

Paris : L'Harmattan, 2009, 202 p.

1 Si la catégorie même de « jeunesse », ou le brouillage de ses frontières avec l'âge adulte, fait en France l'objet de débats, les recherches sur l'entrée dans l'adolescence ne sont pas légion, malgré un intérêt plus marqué pour la population des moins de quinze ans lors de ces dernières années. Il existe alors une distance entre les préoccupations sociales et médiatiques qui en parlent abondamment, la plupart du temps en termes de risques ou de problèmes, et les chercheurs, qui ne les considèrent que sous des angles précis, par exemple comme des élèves, des usagers d'équipements culturels ou des déviants. Le parti pris de Céline Metton-Gayon est donc de réduire cette distance en s'intéressant aux "mondes sociaux» des adolescents dont le téléphone portable et Internet sont le centre, à partir du constat que les activités qu'ils génèrent sont massivement présentes chez les 11-15 ans, et en faisant l'hypothèse qu'ils jouent un rôle dans l'affirmation de leur autonomie et dans la structuration de leur identité. Le concept, repris à Anselm Strauss, doit permettre une approche dynamique et contextuelle des usages de ces deux nouveaux médias qui intègre les injonctions sociétales à la réalisation de soi, tout en envisageant comment elles se traduisent dans les coopérations entre acteurs. Pour mener à bien son étude, cette chercheuse a réalisé 76 entretiens, majoritairement auprès d'adolescents de 11 à 15 ans, mais aussi avec des parents, pour 17 d'entre eux, et avec des frères et sœurs, pour 5 d'entre eux. Sa 
situation d'aide éducatrice dans un collège et d'animatrice de centre de vacances a été la matrice de cette étude, lui permettant d'aborder comme observatrice le monde juvénile; elle a aussi pu analyser des blogs et avoir connaissance de messages SMS. Les résultats de l'enquête sont présentés en trois grandes parties et huit chapitres, qui permettent d'aborder successivement ce que font le téléphone et Internet à la vie en famille des adolescents, puis à la sociabilité juvénile en général, et enfin à la construction des identités sexuées et à la vie sentimentale.

2 La première partie pose d'abord la question de l'accès à ces équipements et à leur usage, un accès qui fait l'objet de négociations et d'accords socialement situés dans la famille. Si les parents partagent la double préoccupation du coût (surtout en matière de téléphone) et de la préservation du temps des devoirs scolaires, les plus socialement favorisés d'entre eux mettent également en avant des arguments beaucoup plus culturels. Le téléphone portable est pour eux l'objet de davantage de méfiance qu'Internet, même si les adolescents savent jouer finement sur les inquiétudes parentales - le téléphone portable permet d'être joint et donc d'être surveillé à tout moment - pour emporter la décision. En matière de pratiques, il est clair que ces deux équipements permettent à la fois de se séparer de la famille, par la construction d'un quant-à-soi privé et de communiquer avec elle, y compris, mais pas seulement, par la fameuse "rétro-socialisation" due à une familiarité plus grande - plutôt qu'à des compétences explicites - avec ces équipements. Face à des préoccupations de contrôle partagées par tous les parents, mais plus intrusives et axées sur la rentabilité scolaire chez les parents de couche moyenne, les adolescents défendent l'accroissement d'autonomie permis par ces nouveaux équipements.

3 La deuxième partie envisage les rapports avec la vie juvénile, en montrant dans un premier temps comment le téléphone surtout, dans ses aspects très matériels, mais aussi les communications sur Internet, sont pris dans les jeux de conventions très fortes déjà observés par ailleurs : codes stylistiques, classements internes où la « bonne maturité » et la bonne distance aux adultes, en particulier à l'école, sont décisives, d'où la double condamnation du " bébé » et du «bouffon ». Mais ils introduisent malgré tout certaines transformations: la popularité recherchée y est davantage possible à objectiver (nombre d'amis et de contacts); les liens faibles y deviennent aussi importants que les liens forts du groupe restreint dans la construction d'un véritable capital relationnel, la mise en scène de la popularité voit s'enrichir ses possibilités d'expression. Surtout, c'est là l'objet d'un deuxième chapitre, les adolescents gagnent des marges d'autonomie relationnelle, y compris à l'intérieur des groupes de pairs bien constitués : grâce au chat, on peut élargir ses horizons ; grâce à l'anonymat, on peut essayer d'autres registres relationnels tout aussi bien que confirmer ses liens habituels en « délirant » avec ses pairs familiers.

Enfin, à l'intérieur de cette problématique, celle des rapports entre les sexes justifie une approche spécifique, évidemment à la hauteur de sa centralité pour les adolescents, mais aussi des évolutions apportées par l'extension de ces modes de communication. Un des intérêts de l'enquête est ici d'élargir à l'ensemble des adolescents des constats faits souvent dans le seul milieu populaire ou dans les quartiers. La communication entre garçons et filles est difficile, tendue et acquiert sur Internet de nouvelles possibilités d'expression de conflictualités, en même temps que de sentiments amoureux, même s'ils sont parfois ensuite difficiles à assumer dans la réalité. Évidemment plus inquiétantes d'un point de vue adulte, les possibilités de 
s'informer sur la sexualité croissent avec la fréquentation plus facile de toutes sortes de sites et d'images (pas uniquement pornographiques), en même temps qu'avec l'opportunité intéressante d'aller «infiltrer » l'autre camp, en changeant, pseudoaidant, d'âge ou de sexe. En qualifiant ces pratiques de "carnavalesques", la chercheuse nuance cependant le propos: les échanges sexuellement très crus ou les travestissements provisoires sont des démesures partielles, qui n'empêchent pas la vraie vie sentimentale de suivre un cours évidemment plus hésitant et pudique. La construction de l'identité sexuée est le lieu d'une complexification, où les provocations entre sexes voisinent avec des socialisations sentimentales diverses. Au total, les mondes sociaux adolescents se voient densifier par Internet et le téléphone portable, rendant le travail éducatif plus complexe mais ne signifiant pas, aux yeux de l'auteure, une intrusion brutale dans le monde adulte. Au contraire, c'est l'adolescence comme espace propre qui se voit ici réaffirmée et enrichie de nouvelles possibilités identitaires. Très agréable à lire, en particulier en raison de son économie d'ensemble et de sa structuration en chapitres nerveux, le livre tient amplement son pari et vient dresser un tableau équilibré et nourri des univers adolescents à l'heure des nouveaux médias. On peut interroger sans doute le choix d'exclure l'école ou les activités institutionnelles proposées aux jeunes de l'enquête, alors même que leur prise en compte aurait sans doute permis de construire plus globalement ces mondes sociaux contre la division du travail entre sociologues, très justement mise en question au début de livre, mais aussi parce que les conditions de l'enquête (en milieu scolaire ou en centre de vacances) paraissaient le permettre. Mais au fond, l'école est malgré tout présente : le contrôle des familles se fait largement au nom de la réussite scolaire et la maturité adolescente s'y codifie largement ; par ailleurs, une enquête doit toujours faire des choix. On peut aussi remarquer la difficulté à faire des différenciations marquées à l'intérieur même des expériences juvéniles, dans la deuxième partie du livre, en dehors de celles que permet la variable de genre. Puisque se jouent dans ces usages des confirmations ou des invalidations partielles de soi, l'adolescent au faîte de sa popularité numérique utilise-til ces outils de la même manière que ceux qui en font un jardin secret ou qui connaissent des expériences difficiles de rejet ou d'invalidation de soi ? Le livre donne une idée de ces différences sans parvenir à les formaliser vraiment, ce qui ne l'empêche pas d'être stimulant et convaincant.

\section{AUTEURS}

\section{ANNE BARRÈRE}

CERLIS, université Paris-Descartes-Paris 5 